\title{
LA CADENA GLOBAL DE VALOR: CONSIDERACIONES DESDE EL CICLO DEL CAPITAL
}

\author{
Seyka Sandoval*
}

Fecha de recepción: 21 de octubre de 2014. Fecha de aceptación: 15 de abril de 2015.

\section{RESUMEN}

El objetivo es analizar la cadena de valor desde la perspectiva de la metodología del ciclo del capital, un ejercicio que permita revisar críticamente los fundamentos a partir de los cuales se establece a la cadena de valor, como el escenario para participar de los beneficios globales de la producción y circulación de mercancías. Se introduce la hipótesis de que la innovación es la causa fundamental de la interdependencia entre capitales y condición necesaria del proceso de valorización. En este sentido, también sería la causa en última instancia de la gobernanza.

Palabras clave: cadenas de valor, ciclo del capital, producción y circulación de mercancías, gobernanza, innovación.

Clasificación JEL: E22, G34, O14.

\section{Global Value Chains: Considerations From the Capital CyCle}

\begin{abstract}
The objective of this article is to analyze the value chain from the perspective of the capital cycle approach, an exercise that allows us to critically review the foundations upon which the value chain is based as a way to share in the global benefits of the production and circulation of commodities. This paper introduces the hypothesis that innovation is the fundamental cause behind the interdependence of capitals and the necessary precursor to the securitization process. In that sense, it would also be the ultimate cause of governance.
\end{abstract}

Key Words: Value chains, capital cycle, production and circulation of commodities, governance, innovation.

\footnotetext{
* Becaria posdoctoral del Instituto de Investigaciones Económicas de la unam. México. Correo electrónico:scsv9@hotmail.com
} 


\section{LA CHAÎNE GLOBALE DE VALEUR: CONSIDÉRATIONS DEPUIS LE CYCLE DU CAPITAL}

\section{Résumé}

L'objectif est d'analyser la chaîne de valeur sous l'angle de la méthodologie du cycle du capital, un exercice qui permette de réviser de manière critique les fondements à partir desquels s'établit la chaîne de valeur, de même que l'espace pour participer aux bénéfices globaux de la production et circulation de marchandises. On introduit l'hypothèse selon laquelle l'innovation est la cause fondamentale de l'interdépendance entre capitaux et une condition nécessaire du processus de valorisation. Dans ce sens, ce serait aussi la source en dernière instance de la gouvernance.

Mots clés: chaînes de valeur, cycle du capital, production et circulation de marchandises, gouvernance, innovation.

\section{A CADEIA GLOBAL DE VALOR: CONSIDERAÇÓES DESDE O CICLO DO CAPITAL Resumo}

O objetivo é analisar a cadeia de valor desde a perspectiva da metodologia do ciclo do capital, um exercício que permita revisar criticamente, os fundamentos a partir dos quais se estabelece a cadeia de valor, como palco para participar dos lucros globais da produção e circulação de mercadorias. Assim se introduz a hipótese de que a inovação é a causa fundamental da interdependência entre capitais e condição necessária do processo de valorização. Neste sentido, também seria a causa em última instância da governança.

Palavras-chave: cadeias de valor, ciclo do capital, produção e circulação de mercadorias, governança, inovação.

\section{从资本循环角度看全球价值链}

\section{摘要:}

本文旨在从资本循环角度分析价值链。价值链是分享商品生产和流通的全球利益的 渠道, 该分析将使我们以批判的眼光看待价值链成为上述渠道的理论基础。本文提 出以下假说: 创新是资本相互依赖的主要原因, 也是证券化过程的必要条件。在这 个意义上，创新也是企业管理最重要的主导因素。

关键词：价值链, 资本循环, 商品生产和流通, 管理, 创新 


\section{INTRODUCCIÓN}

El estudio de las cadenas de valor es una materia reciente ${ }^{1}$ en el diálogo académico, y se centra particularmente en la organización espacial de los capitales con arreglo a la producción de una mercancía o servicio, en un contexto de competencia interdependiente. Recientemente se ha dilucidado que dicha organización de los capitales se explica a partir de la categoría de gobernanza, la cual define una estructura, más horizontal, más vertical, que explica la participación en la ganancia total producida por los capitales involucrados en la cadena. A partir de este planteamiento se ha establecido que la estrategia de desarrollo de capitales y países, debe considerarse en función de la gobernanza de la cadena o las cadenas en las cuales dicho capital y/o país desea participar. Esto es, observar los lineamientos establecidos por el líder de la cadena como condición de competitividad. En este contexto la existencia de la gobernanza es justificada sobre el control de activos estratégicos que permiten posiciones relativas de monopolio/monopsonio.

La importancia de lo anterior radica en dos sentidos. El primero es la consideración de nuevos referentes en el estudio del desarrollo, ${ }^{2}$ y el segundo tiene que ver con la influencia que estos nuevos planteamientos tienen de hecho en la conducción de estrategias y política económica.

Organismos internacionales como la Organización Mundial de Comercio (OMc), la Conferencia para el Comercio y el Desarrollo de las Naciones Unidas (UNCTAD), la Organización para la Cooperación y el Desarrollo Eco-

"The term 'commodity chain' dates from a 1977 article by Terrence Hopkins and Immanuel Wallerstein which appeared in Review, a journal published by the Fernando Braudel Center" (Bair, 2005: 154). En esta misma línea, en 1994, se publicó "Commodity Chains and Global Capitalism. Edited by Gary Gereffi and Miguel Korzeniewicz, this volume contained a number of papers that had been presented at the 16th annual conference on the Political Economy of the World-System, which took place at Duke University in April 1992" (Ibidem). Por su parte, Michael Porter (1986) introdujo la herramienta relacionada con la cadena de valor desde la teoría de la competitividad internacional.

Existen diferentes desarrollos de las cadenas de valor y/o mercancías a la fecha (www.globalvaluechains.org), sin embargo, en esta publicación, consideraremos la evolución del marco analítico desde la perspectiva de la Escuela de Duke, liderada por Gary Gereffi. Lo anterior, no sólo porque el autor es el más citado en el tema, sino porque su propuesta académica ha influido decisivamente en organismos estratégicos para la conducción de la política económica (http://www.cggc.duke.edu/ projects.php, http://www.cggc.duke.edu/pdfs/CGGC-Reports-Pubs-Presentations.pdf).

Por desarrollo se entiende acumulación de capital en un contexto de distribución de las ganancias que estimule economías de escala, que contribuyan a extender e intensificar el proceso de acumulación (Marx, 1976; Pérez, 2004 ). 
nómicos (OCDE), el Fondo Monetario Internacional (FMI), el Banco Mundial (вM), el Foro Económico Mundial (FEM), bancos, fundaciones, organizaciones, así como algunas de las economías líderes y en desarrollo, investigan, crean e implementan de manera decidida, manuales, indicadores y estrategias de política, ligadas a requerimientos que economías y empresas deberán aplicar, en orden de participar y avanzar en las cadenas de valor, estructura de la globalización a partir de la cual se organiza el comercio de manera creciente. $^{3}$

Las implicaciones de lo anterior son variadas, en general, pueden resumirse en la preocupación sobre la precisión de un planteamiento novel, que pretende dirigir las acciones de política pública y privada de países y firmas. Las diversas exploraciones que académicos y hacedores de política realicen sobre el tema, no podrían alegarse suficientes, considerando el alcance del marco analítico bajo discusión.

En este trabajo en particular, el objetivo es analizar la cadena de valor desde la perspectiva de la metodología del ciclo del capital, un ejercicio que permita revisar críticamente, los fundamentos a partir de los cuales se establece a la cadena de valor, como el escenario para participar de los beneficios globales de la producción y circulación de mercancías.

Se eligió la metodología del ciclo del capital porque permite analizar en sus aspectos abstractos, a la cadena de valor como un todo, más allá de la mera articulación de actividades autónomas en torno a un valor de uso. A partir del ciclo podemos encontrar el origen en la creación y distribución del valor y la ganancia en un sistema como la cadena de valor, y analizar la viabilidad de las sugerencias de política, que se supone permite un incremento en la participación de la ganancia de los capitales involucrados. ${ }^{4}$

Se introduce la hipótesis de que la innovación es la causa fundamental de la interdependencia y condición necesaria del proceso de valorización. En este sentido, también es la causa en última instancia de la gobernanza. ${ }^{5}$

https://www.wto.org/english/res_e/statis_e/miwi_e/miwi_e.htm, http://www.oecd.org/sti/ind/ global-value-chains.htm, http://unctad.org/SearchCenter/Pages/Results.aspx?k=global\%20 value\%20chains, http://www.imf.org/external/np/pp/eng/2013/082613.pdf de este documento es utilizar el concepto marxista del ciclo del capital, como herramienta de análisis del objeto de estudio, que en este caso es la cadena de valor. Se propone una discusión-diálogo entre diferentes enfoques teóricos que avancen en la comprensión del fenómeno, ahora global, de la producción y distribución de mercancías y servicios.

5 La innovación es considerada aquí como toda nueva combinación (Schumpeter, 1912, 1977) que permite un incremento en la productividad tal, que conduce al capital en cuestión a producir en las 
En el primer apartado se describe la metodología a partir de un ejemplo analítico, en el que intervienen una muestra de tres capitales, cuya interacción es útil para demostrar cualitativamente la hipótesis sugerida. Los planteamientos aquí realizados se basan en las aportaciones de Carlos Marx y J.A. Schumpeter. Se logró establecer una estructura analítica que muestra al lector la concepción general de la división del trabajo y la innovación, ambos resultados, en segundo y primer orden respectivamente, de la competencia en la nivelación de la tasa de ganancia. ${ }^{6}$

En la segunda parte del documento se discute la definición de gobernanza y sus implicaciones, poniéndolas a prueba en un breve ejemplo que muestra algunos de sus retos. Posteriormente se le observa a la luz de la metodología del primer apartado y se establece el argumento en coherencia con la hipótesis ya mencionada.

Si bien los alcances del documento son de carácter general e introductorio, la participación en el diálogo que sugiere lleva a cuestionar aspectos centrales del concepto de gobernanza, justificado por la existencia de segmentos monopólicos, que aparecen sin explicación más allá de la propiedad de activos específicos, y que la hipótesis aquí planteada resuelve con la introducción de la innovación; por otro lado, discute la existencia y validez de los requerimientos establecidos por las firmas/empresas/corporaciones líderes como condición de participación y ascenso en la cadena de valor.

Se asumen sin cuestionamientos los planteamientos de la crítica a la economía política, como base de herramienta de análisis, no como expresión de dogmatismo, sino de una decisión práctica. Debe establecerse en primer lugar el argumento de la crítica al fenómeno de las cadenas de valor, para ello es necesario partir de bases que son así consideradas como modelo, esperando

mejores condiciones, permitiéndole de esta manera, apropiarse de una cuota de mercado creciente -en posición relativa de monopolio/monopsonio-. Dicha innovación se encontrará, dado el contexto institucional vigente, protegida por derechos de propiedad, a partir de los cuales, se establecen vínculos - de dependencia, competencia o complementariedad- que decantan en las estructuras de gobernanza (Gereffi, Humphrey, Sturgeon, 2005). Por lo anterior, se considera a la innovación como causa de la gobernanza en última instancia.

Si se observa a la cadena de valor en su aspecto concreto, en términos de valor de uso, la complementariedad de actividades concretas en la producción de una mercancía como objetivo de la cadena, podría interpretarse como ausencia de competencia. Lo anterior siendo cierto en primera instancia y considerando el carácter específico de la producción, se revela como inexacto cuando utilizamos el ciclo del capital en su forma dineraria, como forma general. Capitales complementarios se muestran en competencia ante el proceso de distribución de la ganancia producida a lo largo de la cadena en cuestión. 
que el seguimiento del tema permita complejizar dichas bases eventualmente. Al final se presentan las conclusiones.

\section{EL CICLO DEL CAPITAL: METODOLOGÍA}

El ejercicio explicativo que se lleva a cabo está basado en la metodología del ciclo del capital que se encuentra en El capital, de autoría de Carlos Marx (1876 tomo II, secciones primera y segunda) y aspectos relativos a la ganancia y la formación de precios (tomo III, sección segunda), así como de las aportaciones de J.A. Schumpeter en lo referente al tema de innovación en su obra Teoría del desenvolvimiento económico (1912) y Ciclos económicos (1939). En este apartado el objetivo es mostrar cualitativamente la interdependencia creciente en la división del trabajo entre capitales como consecuencia de la innovación.

El ciclo del capital es el proceso de metamorfosis que caracteriza la producción y circulación de mercancías y servicios, como condición necesaria del proceso de valorización y realización, que explica el ciclo de acumulación del capital como motor del sistema económico, ${ }^{7}$ su fórmula general es:

$$
\text { D - FT, MP - ...P...M' - D' }
$$

Donde D es dinero, FT es fuerza de trabajo, MP medios de producción, P capital productivo, M' capital mercantil y D' capital dinerario (Marx, 1976, tomo II, sección 1: 29-57).

Supongamos como punto de partida un solo ciclo de capital, es decir, la producción de una sola mercancía. Imagine una cantidad de dinero inicial, Do, y dos sujetos económicos, uno que posee Do y otro que sólo posee su fuerza de trabajo y la vende libremente, esto es Do-FT (compra de fuerza de trabajo que es al mismo tiempo FT - Do, venta de fuerza de trabajo; aquí habrá que suponer con fines prácticos que el trabajador gasta todo su ingreso en

\footnotetext{
A lo largo del planteamiento más general que contiene la metodología que aquí utilizamos, se seńala repetidamente la idea de que es la apropiación del excedente lo que motiva los actos de los capitalistas. "El capitalista produce la mercancía no por la mercancía misma, no por su valor de uso ni para su consumo personal. El producto que interesa en realidad al capitalista no es el propio producto palpable, sino el excedente de valor del producto por encima del valor del capital consumido en él (Marx, 1976, tomo III, sección 1:47). El "ciclo del capital dinerario...expresa que el fin último y determinante del movimiento es el valor de cambio y no el valor de uso...El proceso de producción se presentan como el eslabón intermedio inevitable, como el mal necesario para alcanzar el objetivo: hacer dinero" (Ibidem, tomo II, sección 1: 64).
} 
consumo), además imagine que el poseedor de Do ha descubierto una nueva combinación ${ }^{8}$ para producir la mercancía Mo, a esto le llamáremos innovación endógena, ${ }^{9}$ que se concretará en MP. Como resultado tenemos el ciclo de Do en la producción y circulación de Mo’:

Do-FT, MP...P...Mo' - Do'

Mo' es el resultado y síntesis del proceso de valorización ${ }^{10}$ de Do. Mo', siguiendo la metodología señalada, tiene un valor mayor que la suma de sus partes dada la característica peculiar de FT de producir valor al ser consumida productivamente. Suponemos que al ser vendida, Mo' se realiza, es decir, repone el valor de sus elementos constitutivos (FT y MP) más el excedente o plusvalor generado, y vuelve a su forma inicial en el ciclo, ya no como dinero, sino como capital dinerario Do'. En este contexto, la venta de Mo' implica, dialecticamente, su compra. Supondremos así que dicha compra es la transacción entre el poseedor de Mo' y los capitalistas y trabajadores, quienes la adquieren para su consumo personal; Mo' abandona en su forma concreta la esfera de la circulación, realizandose. El resultado es el reinicio del ciclo:

"La puesta en práctica de nuevas combinaciones supone, por tanto, simplemente el empleo distinto de las existencias de medios productivos del sistema económico..." (Schumpeter, 1997: 78) "cualquier 'forma de hacer las cosas de manera diferente' en la esfera económica, son todos ejemplos de lo que entendemos con el término innovación" (Schumpeter, 2002: kindle).

9 Definiré como innovación endógena, a la nueva combinación MP, que se introduce al proceso de acumulación a través del mismo capital que la emplea como medio de producción... "No es esencial que la nueva combinación se realice por las mismas personas que controlan el proceso productivo o comercial que debe desplazarse por el nuevo -aunque puede suceder -. Por el contrario, las nuevas combinaciones suelen tomar cuerpo en nuevas empresas que generalmente no surgen de las antiguas, sino que comienzan a producto a su lado" (Schumpeter, 1997: 77).

La innovación (genérica o específica) que permite producir en las mejores condiciones, le otorga a quien la controla -derechos de propiedad- la capacidad de monopolizar relativamente segmentos estratégicos en la producción de ganancias, logrando la apropiación de una "mayor" proporción de éstas en el proceso de distribución, retrasando con ello la nivelación de la tasa de ganancia a través de la competencia.

"La mercancía se convierte en capital mercantil como forma de existencia funcional del valor de capital ya valorizado, surgida directamente del proceso de producción...toda mercancía por el sólo hecho de serlo sería elemento de un capital mercantil" (Marx, 1976, tomo II, sección 1: 45). "M'expresa una relación de valor, la relación entre el valor del producto en mercancías y el del capital gastado en su producción; es decir que expresa la composición de su valor, formado por valor de capital y plusvalor" (Ibidem, p. 47). 
Do' - FT, MP...P...Mono $-\mathrm{Do}^{2}$

Se han sustituido las comillas con número naturales para indicar las rotaciones del ciclo, es decir, el número de veces que el capital en cuestión se ha realizado. ${ }^{11}$ Supongamos ahora, que en este segundo recorrido, MP, la innovación endógena en el ciclo de Do se agotó, y es sustituida por una innovación exógena, ${ }^{12}$ por otro MP introducido en el tercer recorrido de Do, una nueva innovación denominada por M1':

$\mathrm{Do}^{2}-\mathrm{FT}, \mathrm{M1} 1^{\prime} \ldots \mathrm{P} . . . \mathrm{Mo}^{3}-\mathrm{Do}^{3}$

M1' tienen una doble función, es medio de producción en el ciclo de $\mathrm{Do}^{2}$, y el resultado del proceso de valorización -capital mercantil- en el ciclo de D1, la inversión que ha introducido la innovación que ha sustituido a MP en el ciclo de Do, incrementando la división del trabajo entre capitales: ${ }^{13}$

D1 - FT, MP...M1' - D1'

El proceso de realización en D1, la venta $\left(\mathrm{M} 1^{\prime}-\mathrm{D} 11^{\prime}\right)$, es al mismo tiempo la compra $\left(\mathrm{Do}^{2}-\mathrm{M} 1^{\prime}\right)$, por lo tanto $\left(\mathrm{D} 1^{\prime}=. \mathrm{xDo}^{2}\right)$ donde. $\mathrm{x}$ es parte propor-

11

"El tiempo de rotación de un capital dado es igual a la suma de su tiempo de circulación más su tiempo de producción. Es el espacio de tiempo que se extiende desde el momento en que se adelanta el valor de capital bajo una forma determinada hasta el retorno del valor de capital en proceso bajo la misma forma" (Marx, 1976, tomo II, sección 2: 183).

12

La innovación exógena es la nueva combinación que se introduce a través de un capital diferente al que la utiliza como medio de producción. En la interacción entre dos o más capitales, la definición entre innovación exógena y endógena es relativa. En nuestro esquema es endógena para el que la introduce y la emplea como medio de producción, y exógena para que el que sólo la emplea como medio de producción, pues ha sido introducida por un capital diferente. Pero si damos un paso atrás y separamos la generación de las innovaciones de las tareas del poseedor de Di, este sería un proceso exógeno en cuanto a invención se refiere y endógeno como elemento del proceso cíclico de acumulación de capital, "Si bien los empresarios [agentes económicos que llevan a la práctica la nueva combinación o innovación] pueden ser inventores como pueden ser capitalistas, lo son por coincidencia y no por naturaleza, y viceversa. Además, las innovaciones que llevarán a la práctica los empresarios, no precisan ser invenciones en forma alguna...el liderazgo del empresario conduce los medios de producción a nuevos caminos (Schumpeter, 1997: 98).

"Aquí MP y FT sólo se diferencian en que MP en manos de su vendedor puede ser = M', es decir, puede ser capital cuando MP es la forma mercantil del capital de dicho vendedor, mientras que FT para el obrero siempre es sólo mercancía y no se convenirte en capital más que en manos del comprador, como parte constitutiva de P”. (Marx,1976, tomo II, sección 1: 103). 
cional de $\mathrm{Do}^{2}$, ya que $\mathrm{xDo}^{2}$ fue invertida en FT, M1' y en el consumo capitalista. Aquí, el consumo de M1', a diferencia de $\mathrm{Mo}^{\prime}$ y $\mathrm{Mo}^{2}$, es productivo, ejerciéndose en un ciclo diferente.

Los ciclos de los capitales $\mathrm{Do}^{2}$ y D1 son ahora interdependientes, ello estriba en que para que $\mathrm{Do}^{2}$ adquiera M1' a través de la compra, como elemento del proceso de valorización, D1 debe realizar M1' a través de la venta. Así la compra, vehículo del proceso de valorización, es simultáneamente, la venta, acto de realización.

Si se repite el supuesto de la introducción de una innovación, ahora en el ciclo de D1, las cosas se presentan así:

D1'-FT, M2'...P...M12 $-\mathrm{D}^{2}$

MP, la innovación endógena en D1, ha sido sustituida en la segunda rotación por M2', implicando con ello una revolución en la forma de producir $\mathrm{M1}^{2}$. Es decir, M1' y $\mathrm{M1}^{2}$ como resultados separados en el tiempo del ciclo de D1, expresan la evolución en el ciclo a través de la innovación. En lo general $M 1^{\mathrm{n}}$ es siempre el resultado del proceso de valorización de D1 ${ }^{\mathrm{n}}$; en lo particular su expresión concreta se modifica a partir de los cambios que la innovación introduce en su producción y circulación.

M2' sería el resultado de:

D2 - FT, MP...P...M2'-D2', así D2' = .xD1', o bien

$\mathrm{D} 2^{\prime}=. \mathrm{x}\left(. \mathrm{xDo}^{2}\right)=. \mathrm{x}^{2} \mathrm{Do}^{2}$, porque $\mathrm{D} 1^{\prime}=. \mathrm{xDo}^{2}$

D2 es aquí un nuevo capital que se integra a la división del trabajo a partir de la introducción de una nueva mercancía M2', que es definida como innovación exógena en D1'. La interdependencia se ha ampliado en la medida en que el resultado del proceso de valorización de D2, es decir M2', es simultáneamente medio de producción en el proceso de producción en el ciclo D1'-FT, M2'...P...M12 ${ }^{2}$, el cual a su vez, tiene como resultado del proceso de valorización $\mathrm{M1}^{2}$, el medio de producción que sería utilizado en el proceso de producción en el ciclo de $\mathrm{Do}^{3}$.

Este recorrido de condicionamientos en su forma material, procesos de producción-valorización dependientes entre sí, se justifica ante la tendencia creciente en la división del trabajo, que expresa límites al espacio de reproducción y realización cada vez más amplios, gracias a la presencia de la innovación. 
Tan pronto como la producción por medio del trabajo asalariado se generaliza, la producción mercantil debe ser la forma general de la producción. Partiendo del supuesto de que ésta es la forma general, ella condiciona, por su parte, una división siempre creciente del trabajo social, es decir, una especialización siempre mayor del producto que un capitalista determinado produce como mercancía, una separación cada vez mayor de procesos de producción complementarios que se vuelven autónomos (Marx, 1976, tomo II, sección 1:42).

Esta interdependencia, sin embargo, trasciende los valores de uso que articulan técnicamente los diferentes ciclos del capital, ligando los proceso de realización, que en el esquema presentado se sintetizan así: $\mathrm{D}^{\prime}$ ' = .xD1', o bien $\mathrm{D} 2^{\prime}=. \mathrm{x}\left(. \mathrm{xDo}{ }^{2}\right)=. \mathrm{x}^{2} \mathrm{Do}^{2}$.

La realización de D2, es decir la venta M2' - D2' es al tiempo la compra de $\mathrm{D} 1$ ' - M2', una parte de $\mathrm{D} 1$ ' (.xD1') invertida en medios de producción M2', por tanto la realización de D2 está en función de la realización de D1, así $\left(\mathrm{D} 2{ }^{\prime}=. \mathrm{xD} 1{ }^{\prime}\right)$ y para que $\mathrm{D} 1$ se realice, otra venta debe ocurrir, $\mathrm{M1}$ ' - D1' que a su vez es la compra $\mathrm{Do}^{2}-\mathrm{M1}^{\prime}$, y como no es posible comprar si no se ha vendido, la realización de $\mathrm{D} 1$ estará en función de la realización de $\mathrm{Do}^{1}-\mathrm{Mo}^{2}-\mathrm{Do}^{2}$, que se logra a través del consumo de capitalistas y trabajadores. Al ocurrir lo anterior, el ciclo $\mathrm{Do}^{2}$ se reinicia adquiriendo FT y M1', realizando con ello a D1' (M1' - D1') y permitiendo a la vez que D1' se reinicie con la compra de FT y M2' realizando así a D2 (M2' - D2'). Esta concatenación permite que, esquemáticamente, $\mathrm{D} 1^{\prime}$ pueda ser sustituido por . $\mathrm{xDo}^{2}$ en $\mathrm{D} 2^{\prime}=. \mathrm{xD} 1^{\prime}$, ya que $\mathrm{D} 1^{\prime}=. \mathrm{xDo}^{2}$, así, $\mathrm{D} 2^{\prime}=. \mathrm{x}\left(. \mathrm{xDo}{ }^{2}\right)=. \mathrm{x}^{2} \mathrm{Do}^{2}$, comprobando la interdependencia en la realización del valor entre los capitales involucrados. ${ }^{14}$

El lector puede advertir que nuestro esquema inicia con un ciclo que tiene como destino de su producto, el consumo personal de capitalistas y trabajadores; y que dicho consumo es en última instancia lo que permite que sucedan la suma de las interacciones en la realización de los capitales involucrados. Esto tiene implicaciones que vale la pena mencionar. A primera vista podríamos vernos compelidos a afirmar que el objetivo último y mobile del sistema económico es la satisfacción de necesidades, en la medida en que ello condiciona la reanudación del ciclo económico. No obstante, la satisfacción del consumidor es sólo un vehículo en el proceso de realización, en el cual el aspecto concreto carece de importancia, tal y como se ha establecido bajo la metodología aquí utilizada. Por otro lado, y considerando nuestra hipótesis de introducción periódica de innovaciones, se muestra que son las necesidades del proceso de valorización-realización (sustitución de MP por $\mathrm{Mi}^{\mathrm{n}}$ ) las que estimulan la evolución económica expresada en la innovación. Ésta aparece como un elemento nuevo fuera de la esfera de las necesidades particulares "estas perturbaciones del equilibrio, aparecen en la esfera de la vida industrial y comercial, y no en la esfera de las necesidades de los consumidores...Por lo general, es el productor quien inicia el cambio económico, educando incluso a los consumidores si fuera necesario; les enseña a necesitar nuevas cosas, o cosas que difieran 
Hasta aquí hemos supuesto que cada capital que se integra por primera vez, lo hace a partir de una innovación que funciona como medio de producción en un ciclo diferente; ejemplo, $\mathrm{M1}^{\prime}$ en $\mathrm{Do}^{2}$ y M2' en D1'. Ubicamos estas innovaciones sólo en la sinergia entre FT y MP, que tiene como resultado P, el capital productivo, pues de acuerdo a la metodología aquí utilizada, sólo en este proceso de transformación el capital se valoriza. Este supuesto es importante pues mantiene coherencia con la hipótesis, de que la innovación es condición del proceso de valorización. ${ }^{15}$

Se consideró inicialmente, que las innovaciones son gestaciones endógenas denominadas MP, que se agotan históricamente y son sustituidas; por tanto, cada vez que se introducen, se habla de un salto cualitativo en el proceso de producción a lo largo de un periodo determinado. La justificación de la sustitución se sustenta por dos vías: el agotamiento físico de MP, que hace necesaria su renovación, y las presiones de la competencia ante el surgimiento de medios más productivos -innovaciones -. (Marx, 1976, tomo II, sección 2: 206). Mientras que el remplazo de MP sea consecuencia del desgaste normal del uso, hablaremos de mejora continua, más no de innovación. En nuestro esquema, sólo la competencia logra la sustitución de MP por Mi', es decir, la introducción de una innovación.

Retomando el esquema aquí desarrollado consideramos tres capitales que producen tres mercancías distintas. Los ciclos de Do, D1 y D2 son partes complementarias de un ciclo global, que tiene como resultado una mercancía para consumo final.

Dadas las diferencias en el valor de uso de las mercancías producidas, cada capital tiene una composición distinta entre sus cantidades y valores de fuerza de trabajo -que se denomina capital variable v- y de medios de producción -capital constante c-. Esta composición en su expresión de valor c:v es definida como composición orgánica del capital ${ }^{16}$ (coc). Si recordamos que v tiene

en algún respecto de las ya existentes”. (Schumpeter, 1997: 75-76). La aclaración de este punto es relevante pues podría guiarnos a conclusiones incorrectas en las cuales consideráramos, que sólo la expansión de las necesidades sociales solventes podría sostener la rotación del ciclo del capital, esto no representaría un resultado preciso de acuerdo a la metodología aquí utilizada.

Ello no quiere decir que no existan innovaciones que se sitúen en la esfera de la circulación, acelerando el ciclo de rotación e impactado indirectamente en el proceso de valorización y acumulación. te pasivo, entre los capitales variable y constante...A la composición de valor del capital, en tanto la misma resulta determinada por su composición técnica y la refleja, la denominamos la composición orgánica del capital" (Marx,1976, tomo III, sección 2: 182-184) ver composición técnica del capital y su relación con la composición orgánica del capital en (Ibidem, p. 183). 
la característica de producir valor cuando es consumida productivamente, entonces reconoceremos que las mercancías producidas tienen un valor superior al de sus partes constitutivas, c y v; esto es $\mathrm{Mi}^{\prime}=\mathrm{c}+\mathrm{v}+\mathrm{pv}^{17}$ donde pv es el excedente o plusvalor.

Para el capitalista, que no sólo adelanta como parte de su capital, v sino $\mathrm{c}+\mathrm{v}$, es indiferente la fuente de su excedente o plusvalor, por tanto, calcula éste bajo la noción de la ganancia con base en su capital global adelantado g'= (pv/C) donde C= c+v (Marx, 1976, tomo III, sección 1:49). La tasa de ganancia $(\mathrm{g}$ ') es la proporción del beneficio obtenido sobre un capital $\mathrm{C}$ puesto en funciones (Idem).

No obstante la sustitución que el capitalista hace de pv' por g', las diferencias en la composición de los valores c:v de las mercancías disímiles, se sostiene en nuestro ejemplo. El precio de costo no se modifica si consideramos al excedente como plusvalor o ganancia.

Establecido lo anterior y antes de salir al mercado, cada capitalista debe establecer sus precios, y lo hace considerado sus costos o precio de costo (valor de $\mathrm{c}+\mathrm{v}$ ) y la ganancia esperada. Dicha ganancia se fija a partir de lo que está definido como ganancia media, ${ }^{18}$ la cual expresa el promedio del excedente, en función de la ganancia total, que es producida por los tres capitales participantes y será distribuida entre ellos. En este contexto el establecimiento de los precios en cada caso será igual a $\mathrm{c}+\mathrm{v}+\mathrm{g}^{*}$ donde $\mathrm{g}^{*}$ es ganancia media. El precio así derivado se conoce como precio de producción (pp) el cual expresa la cantidad de dinero a la cual el capitalista esperaría vender su mercancía. En nuestro ejemplo existirán tres precios de producción $\mathrm{pp}_{\mathrm{o}}, \mathrm{pp}_{1}, \mathrm{pp}_{2}$.

"El valor de toda mercancía producida de manera capitalista, $M$, se expresa en la fórmula $\mathrm{M}=\mathrm{c}+\mathrm{v}$ + pv. Si de este valor del producto descontamos el plusvalor, pv, nos queda un mero equivalente o un valor de reposición en mercancía por el valor del capital c+v desembolsado en los elementos de la producción...parte del valor de la mercancía que repone el precio de los medios de producción consumidos y el precio de la fuerza de trabajo empleada, sólo repone lo que le cuesta la mercancía al propio capitalista, y por ello constituye para él el precio de costo de la mercancía” (Ibidem, sección 1: 30) "el excedente sobre el precio de costo, ósea el plusvalor" (Ibidem, p. 37). "El proceso de consumo de la fuerza de trabajo es al mismo tiempo el proceso de producción de la mercancía y del plusvalor" (Ibidem, tomo I, sección 2: 213) más sobre el concepto de plusvalor (Ibidem, sección 3, 4 y 5).

“...diferentes tasas de ganancia resultan niveladas por la competencia en una tasa general de ganancia, que constituye el promedio de todas esas diferentes tasas de ganancia. La ganancia que con arreglo a esta tasa general de ganancia, corresponde a un capital de magnitud dada, cualquiera que sea su composición orgánica, se denomina la ganancia media. El precio de una mercancía, que es igual a su precio de costo más la parte de la ganancia media anual que le corresponde, según la relación de sus condiciones de rotación, sobre el capital empleado para producirla (no sólo sobre el capital consumido para producirla), es su precio de producción (Ibidem, tomo III, sección 2: 199). 
Cuando las mercancías salen al mercado con el objetivo de ser vendidas, los precios de producción se enfrentan con los precios de mercado, ${ }^{19}$ que pueden ser mayores o menores que éstos. Los precios de mercado están definidos prima facie por los movimientos entre la oferta y la demanda.

Si el precio de producción es mayor que el precio de mercado, el capitalista no podrá realizar el valor total de sus mercancías; si por el contrario el precio de producción es menor que el precio de mercado, no sólo se realizará su valor, sino que se obtendrá una ganancia extraordinaria.

Si consideramos un capital individual que "ópera con una productividad mayor que la productividad social media, presentando sus productos con un valor más bajo que el valor social medio de esa mercancía, con lo cual realiza una ganancia extraordinaria" (Ibidem, p. 59). Esperaremos que:

...si alguno produce más barato y puede envilecer más el precio, apropiarse de un mayor volumen del mercado vendiendo por debajo del precio corriente de mercado o del valor de mercado, lo hace, y de este modo comienza la acción que poco a poco obliga a los otros a introducir el modo de producción más barato, el cual reduce a una nueva medida menor el trabajo socialmente necesario. Cuando un bando tiene supremacía, ganan todos cuantos pertenecen a él; todo ocurre como si tuviesen que imponer un monopolio común. Si un bando es el más débil, cada cual podrá buscar, por su propia parte, la manera de ser el más fuerte (por ejemplo, el que trabaja con menores costos de producción) (Ibidem, sección 2: 245).

En este contexto los capitales aspirarán a participar en el mercado en el cual los precios de producción sean menores que los precios de mercado, tratando de emular los medios productivos que causan este fenómeno. Suponiendo

"Cualquiera que sea la manera en que se fijen o regulen los precios de las diversas mercancías entre sí, en primera instancia, es la ley del valor la que rige su movimiento. Cuando disminuye el tiempo de trabajo requerido para su producción [aumento de la explotación del trabajo, aumento de la productividad como consecuencia del surgimiento de métodos más productivos o innovaciones] disminuyen los precios..." (Ibidem, p. 224). En este contexto el precio de venta es el precio al que se intercambia la mercancía en el mercado, esto es un precio por encima del precio de costo (Ibidem, sección 1: 51). No obstante, "Aunque el excedente del valor de la mercancía por encima de su precio de costo se origina en el proceso directo de la producción, sólo se realiza en el proceso de la circulación, y adquiere la apariencia de emanar del proceso de la circulación tanto más fácilmente por cuanto en la realidad, dentro de la competencia, en el mercado real, depende de las condiciones del mercado el que ese excedente se realice o no, y en que grado" (Ibidem, sección 1: 50). Situados ya en la esfera la circulación "los dos límites de su precio de venta son: por un lado, el precio de producción de la mercancía, acerca del cual no decide; por otro lado, la tasa media de ganancia, que es algo que tampoco depende de él (Ibidem, sección 4: 392). 
que esto es posible, el efecto es un incremento en la oferta en el mercado en cuestión, que reducirá el precio de mercado acercándolo al precio de producción, provocando la desaparición de la ganancia extraordinaria; de continuar la tendencia incluso podría reducirse o desaparecer la ganancia media, si los precios de mercado continúan cayendo.

En este escenario, la búsqueda por espacios en los que los precios de producción sean menores que los precios de mercado impulsan la innovación. La cual reduce el tiempo de trabajo necesario aumentando el trabajo excedente, incrementando la tasa de explotación y productividad, que temporalmente arrojara una ganancia extraordinaria.

Podemos concluir este apartado establecido que la interdependencia es el resultado de la división del trabajo entre capitales, caracterizada por la sustitución de MP por $\mathrm{M}^{\mathrm{n}}$, y que esta división tiene como base material la introducción de innovaciones a partir de la dinámica de la competencia. Concretamente, la interdependencia se muestra en la relación inter ciclo (que puede ser inter firma, al interior de una firma entre sus departamentos) o inter industria (entre firmas/empresas de una misma rama de producción o industria) expresando el condicionamiento mutuo entre los procesos de valorización y realización, a través de la incorporación $\left(\mathrm{D}^{\mathrm{n}}-\mathrm{M}^{\mathrm{n}}\right.$, FT... P... $\mathrm{M}^{\mathrm{n}+1}$ $-\mathrm{D}^{\mathrm{n}+1}$ ) y producción (Di - FT, MP... P... $\mathrm{M}^{\mathrm{n}}-\mathrm{D}^{\mathrm{n}}$ ) de la innovación como condición de la existencia de la ganancia extraordinaria.

\section{GOBERNABILIDAD Y DESARROLLO}

El ejemplo analítico presentado en el apartado anterior, pretendió exponer de manera general y breve, el proceso en el cual los capitales producen, circulan y compiten a través de la innovación en el marco del ciclo del capital. En el plano en el que fue expuesto, el circuito fluye sin perturbaciones permitiendo que el motor de la innovación, la generación de medios más productivos, se erija como la única fuente de ganancias extraordinarias y liderazgo en el mercado. En un contexto de darwinismo económico, los capitales capaces de producir "en las mejores condiciones" serán los únicos poseedores de la plusganancia o ganancia extraordinaria.

En un nivel de análisis más concreto, habríamos de introducir nuevos supuestos que si bien no contradicen el argumento en general respecto de la innovación, si perturban la distribución de las ganancias extraordinarias. Si se considera que la nivelación de la tasa de ganancia, como producto de la difu- 
sión ${ }^{20}$ de la innovación, es más rápida en la medida en que el capital y la fuerza de trabajo incrementen su movilidad, lo contrario es cierto, todo aquello que reduzca la movilidad del capital y la fuerza de trabajo, aumenta la desigualdad en las tasas de ganancia (Marx, 1976, tomo III, sección 2: 247). Por lo tanto, los capitales sólo podrán incrementar su participación en la ganancia total, ${ }^{21}$ si aquellos capitales que producen en las mejores condiciones reducen sus posiciones de monopolio, permitiendo la libre difusión de la innovación a través de la competencia. $\mathrm{O}$ bien, los capitales que no producen en las mejores condiciones, introducen innovaciones que les permitan hacerlo desplazando a aquellos que ostentan el liderazgo. En este contexto, se analiza la cadena de valor como la estructura organizacional del capital a partir de dicho liderazgo. ${ }^{22}$

20

Entenderemos por difusión el proceso por medio del cual la competencia generaliza los medios más productivos. En Marx observamos esto en la nivelación de la tasa de ganancia. En Schumpeter encontramos un proceso similar en cuanto a la aparición creciente del empresario "cuanto más familiar se haga el proceso de desenvolvimiento y en la medida en que llegue a ser cuestión de cálculo para todos los interesados, y cuánto más débiles se hagan los obstáculos en el curso del tiempo, tanto menores serán las cualidades de liderazgo que se requieran para llevar a cabo innovaciones" (1997: 229-230).

21 Si consideramos la metodología propuesta en el apartado primero, la ganancia total es la suma del plusvalor producido a lo largo de todo el proceso del ciclo del capital, es decir, la diferencia entre D y D'. En el planteamiento marxista, en la medida en que se ha establecido que sólo la sinergia entre FT y MP puede producir nuevo valor, este plusvalor expresado en ganancia se origina sólo en el proceso de producción.

En el marco de la cadena de valor, se considera como ganancia total la suma del valor añadido a lo largo de la cadena (Sturgeony Memedovic, 2011). Se supone que cada actividad, añade valor a la mercancía o servicio en cuestión. Independientemente que estas actividades se encuentren en el proceso de producción o circulación de mercancías y servicios. Lo que este documento explora es el análisis de la cadena de valor y la distribución de las ganancias en ella producidas, a partir de la difusión de la innovación como base de la gobernanza, lo cual constituye el supuesto central. Este supuesto cerca, en ambos marcos analíticos, la creación de valor sólo en el proceso productivo, como condición-puente del diálogo académico. La innovación en este sentido debe considerarse sólo en el proceso de producción en términos de la discusión aquí desarrollada.

Las cadenas de valor son uno de los corolarios del proceso de cambio histórico iniciado en los ańos setenta -crisis, revolución informática y neoliberalismo-, es una forma estructural de la globalización que a través del nuevo rol que adquiere la empresa global, asigna actividades económicas en diferentes espacios en función de las ventajas comparativas y competitivas que estos espacios ofrecen. La red que resulta de lo anterior expresa todo el ciclo del capital de diferentes mercancías y servicios, en el que interactúan diversos actores tanto económicos como institucionales. Las cadenas de valor expresan de manera concreta la nueva división internacional del trabajo, en cada uno de los bienes y servicios que se producen alrededor del mundo. La funcionalidad que las redes tienen en el proceso de acumulación se expresa en la eliminación de las barreras que constreñían la libre movilidad del capital. 
La cadena global de valor es una propuesta analítica introducida en la década de los setenta. ${ }^{23}$ Tuvo como carta de presentación más sobresaliente la obra editada por Gary Gereffi y Miguel Korzeniewicz, Commodity Chains and Global Capitalism, publicada en 1994, en la cual se definió como la organización de un conjunto de redes en relación a la producción de una mercancía, involucrando una serie de entidades económicas e institucionales (1994: 2); posteriormente fue considerada como "el amplio rango de actividades involucradas en el diseño, producción y comercialización de un producto" (Gereffi, 2000: 58; 2001a: 1618; 2001: 14). Sus objetivos se plantearon en relación a "cuestiones de reorganización industrial, coordinación, gobernanza y poder en la cadena” (Gereffi et al., 2005a: 168).

Uno de los aspectos más notables de esta propuesta es la noción de gobernanza ${ }^{24}$ introducida en 2005 por Gereffi G., Humphrey J. y T. Sturgeon, la cual plantea un conjunto de cinco formas analíticas de control-coordinación en la red, ${ }^{25}$ ejercidas por la firma líder, implicando con ello un plano asimétrico de poder entre los capitales involucrados en la producción de una mercancía o servicio. El objetivo es que "la teoría de la gobernanza en las cadenas de valor (...) sea útil para generar instrumentos de política eficaces, relacionados con el ascenso industrial, el desarrollo económico, la creación de empleos y el alivio de la pobreza" (Gereffi et al., 2005: 79).

23

Para un mayor desarrollo sobre el tema, y dadas las limitaciones de espacio, se recomienda: Gereffi (2001), Kaplinsky (1998), Bair (2005) y Sandoval (2012).

24

El término gobernabilidad o gobernanza se refiere a las diferentes formas de control/coordinación que ejercen las empresas líderes en una cadena específica. La base de la tipificación considera tres corrientes teóricas: los costos de transacción (Williamson, 1975 citado en Gereff, Humphrey y Sturgeon, 2005: 80) especificidad de los activos, capacidades, la producción en red (Arndt y Kierzkowski, 2001 y Fenestra, 1998 citados en Gereffi, Humphrey y Sturgeon, 2005: 79-80) -integración al comercio, coordinación de la dispersión de actividades, y la capacidad tecnológica y el nivel de aprendizaje de la empresa (Jarillo, 1988; Lorenz, 1988; Powell, 1990 y Thorelli, 1986 citados en Gereffi, Humphrey y Sturgeon, 2005: 80). "Así, nuestra tipología, identifica cinco tipos básicos de gobernabilidad en la cadena de valor. Estos son analíticos, no empíricos, aunque ellos han sido en parte derivados de observaciones empíricas" (Gereffi, Humphrey y Sturgeon, 2005: 83-84).

25

Las formas mencionadas son: mercado, modulares, relacionales, cautivas y jerárquicas. Cada una define un tipo especial de gobernanza, donde aquella de tipo mercado es más horizontal, y aquella de tipo jerárquica más vertical. La mayor o menor verticalidad en la cadena está asociada directamente a la necesidad de coordinación por parte del líder (Gereffi et al., 2005: 83-84). Antes de esta clasificación se había establecido que las cadenas pudieran ser dirigidas por el comprador o por el vendedor haciendo alusión a grandes detallistas o productores intensivos en tecnología como líderes en la cadena de valor (Gereffi, 1994, 2001). 
Comprender la gobernanza es la clave en el ascenso o upgrading, el cual significa "...el proceso por el cual los agentes económicos - naciones, firmas y trabajadores- se mueven de actividades de bajo valor a actividades de alto valor, en las redes de producción global" (Gereffi, 2005a: 171) y está en función de "vínculos selectivos con los diferentes tipos de empresas líderes (lead firms) de las industrias globales" (Gereffi, 2001: 13).

La gobernanza se expresará en la competencia entre capitales y la participación desigual de cada uno en el total de la ganancia producida. "Lo que distingue a las empresas líderes de sus seguidores y subordinados es que controlan el acceso a los recursos más importantes... que generan los ingresos más rentables ${ }^{26}$ de la industria" (Gereffi, 2001: 20; 2001a: 1622).

En el planteamiento de Gereffi et al. (2005) la base de la gobernanza está asociada a la complejidad de las transacciones, codificación de la información y capacidad de los proveedores, ${ }^{27}$ es decir, a la transferencia de información y conocimiento necesaria para realizar una transacción, la medida en la que este conocimiento es codificado y transmitido, y la capacidad de los proveedores actuales y potenciales de satisfacer los requerimientos de la transacción (Gereffi, 2005: 85). Estos aspectos están relacionados con la especificidad de activos y costos de transacción. ${ }^{28} \mathrm{El}$ modelo supone que una especificidad alta de activos, productos con procesos integrales o sensibles al tiempo, incrementan los mundane transaction cost que se definen como los costos de coordinar la cadena de valor (Gereffi, 2005: 84).

La rentabilidad es un concepto similar al de ganancia. El capitalista obtiene su tasa de rentabilidad o de ganancia calculando el excedente sobre el costo de sus medios de producción y su fuerza de trabajo, sin importar el origen específico de tal excedente. No obstante y en función de la metodología aquí utilizada, es necesario aclarar, para futuros desarrollos del tema, que una actividad puede ser rentable sin producir valor. Tales son algunas relacionadas con la circulación de las mercancías, las cuales capturan valor a través del cargo que producen los costos de circulación en la realización del plusvalor.

Esta perspectiva basada en los trabajos del Premio Nobel Oliver Williamson, establecen a la transacción como la unidad económica de análisis. Y definen a ésta como algo que ocurre cuando un bien o servicio es transferido a través de una interfaz tecnológicamente separable (Williamson, 1981). El planteamiento considera como dimensión principal la especificidad de los activos, en relación directa con el nivel de los costos, los cuales conducirán la decisión sobre las actividades que se externalizan e internalizan, definiendo así la organización de la firma. Esta noción es parte central en la propuesta de gobernanza recogida en Gereffi et al., 2005 a partir de los trabajos de Williamson $(1975,1983)$. En este sentido costos de transacción y costos de coordinación se utilizan de manera indistinta. 
La dinámica en el ejercicio de la gobernanza puede sintetizarse en incrementos y decrementos sucesivos de los costos de coordinación. Estos aumentan en la medida en que se introducen nuevas demandas, procesos organizacionales, diferenciación del producto, entre otros, -aumento en la complejidad de transacciones y especificidad de activos- y disminuyen cuando se establecen técnicas y estándares que hacen más eficiente el flujo de información -incremento en la codificación y capacidad de los productores- (Gereffi, 2005: 8485). La combinación de estos aspectos definirá la verticalidad u horizontalidad de la cadena de valor.

$\mathrm{Al}$ introducir la gobernanza sobre la base de un eficiente flujo de información y conocimiento, en función de la especificidad de los activos, que redunde en mayores o menores costos de transacción, Gereffi et al. (2005) ubica el fenómeno del ascenso en el plano organizacional, a través del control sobre activos y procesos nuevos y/o altamente diferenciados. El liderazgo se ejercerá a partir de una configuración de control-coordinación, más vertical, más horizontal, que obedece a la reducción de los costos de coordinación y la maximización de las rentas del líder. Las oportunidades de ascenso para las firmas no líderes estarían concentradas, en general, en reducir dichos costos de transacción/ coordinación por medio de respuestas eficientes ante la información del líder, a cambio de una participación en el valor siempre menor que aquella obtenida por la firma que ejerce la gobernanza. ${ }^{29}$

La cadena global de valor se organiza sobre la propiedad de activos diferenciados que otorgan el control sobre "los segmentos más rentables de la industria" y permiten una posición relativa de monopolio a partir de la cual se convoca la competencia de otros capitales a realizar actividades complementarias. Competencia en la que dichos capitales deben satisfacer el criterio de la mayor eficiencia al menor costo, como condición de reducir al límite los costos de coordinación en la cadena. Es en este escenario que los capitales involucrados deberán desarrollar estrategias de integración y desarrollo.

El planteamiento parece arrojar limitaciones de origen respecto de la consecución del ascenso o upgrading. Si la posición del líder está en función del monopolio sobre los segmentos más rentables, se asume que éste utilizará toda medida posible para mantener dicha posición, por lo tanto, la difusión de aquello que permite la mayor rentabilidad será "protegido" de la competencia de otros capitales, inhibiendo a éstos en su participación en la ganancia total. de la empresa" (Gereffi et al., 2005: 91). 
En segundo lugar, si el ascenso está en función de los vínculos con el líder de la red, dada la monopolización mencionada que impide la competencia directa, éstos sólo pueden establecerse a partir de actividades complementarias, que implican costos de coordinación regulados por medio de técnicas y estándares como condición de competitividad. En conclusión, las posibilidades de desarrollo de los capitales seguidores están directamente relacionadas con el cumplimiento de estos requerimientos.

Desde esta perspectiva se espera que la vinculación con firmas más desarrolladas, ergo más rentables, acercara a los capitales seguidores a estándares "superiores" que redundarán en mayor productividad, ganancias, flujos de inversión y mejores salarios; no obstante los beneficios que ello supone, se advierte, crean diferencias entre capitales integrados y no integrados, afectando el espacio económico que hospeda al capital seguidor en materia de competencia, trabajo y bienestar social (Sturgeon y Memedovic, 2011: 2-4) la solución a ello, está influenciada por las políticas encaminadas a difundir los beneficios derivados de la integración.

Supongamos entonces que un capital i se integra a una cadena de valor que produce una mercancía $x$. En esta cadena el capital líder ostenta una posición monopólica que le permite capturar ${ }^{30}$ el $51 \%$ de la ganancia total generada ${ }^{31}$ en la cadena. El capital i a condición de cumplir estándares obtiene una captura parcial del valor total. El objetivo, sin embargo, es incrementar dicho porcentaje. ¿Cómo lo logra?

Si el $51 \%$ del valor está protegido por la posición de monopolio, sólo puede incrementar su participación compitiendo con el resto de los capitales seguidores, por una parte del $49 \%$ restante. Si se ha establecido que el ascenso se alcanza cumpliendo con los estándares establecidos como condición de reducir los costos de coordinación de la cadena, entonces, aquellos capitales que satisfagan el criterio al menor costo serán los que logren incrementar su participación en la ganancia. Si todos comparten el objetivo, la búsqueda por la reducción de los costos impulsará mecanismos que incrementen el flujo de información, su decodificación y cumplimiento, que resultan en una mayor velocidad en la rotación de toda la cadena. ${ }^{32}$

30

Capturar es diferente de producir, y está relacionado con la distribución de las ganancias más allá del origen de éstas.

31

Aquí cabría la pena aclarar que el capital líder pudiera acaparar más capital que el que genera, sea o no el propietario de la innovación.

32 Menores costos de transacción permiten un flujo con menores perturbaciones en el ciclo del capital, beneficiando la rotación de ésta acelerando el proceso de acumulación y por tanto la suma de las ganancias en un tiempo determinado. 
Si consideramos que el capital i introduce un mecanismos que lo lleva a satisfacer los estándares del líder a un menor costo que sus competidores, y recordamos que sólo el segmento líder es monopólico, la competencia hará que la ventaja del capital i se diluya rápidamente en la medida en que se difunde, nivelando la posición de todos los capitales seguidores y haciendo necesario un nuevo mecanismo que reduzca de nuevo los costos de coordinación, que a su vez, se difundirá obligando a repetir el proceso en un espiral descendente, que conduce a los capitales seguidores a constreñir sistemáticamente sus costos de transacción, como condición de competitividad, y sus precios por medio de la competencia, haciendo analíticamente imposible el salto mientras el segmento más rentable continúa monopólico. En este escenario, la consideración de la cadena de valor como driver of development sería contradictoria si señalamos que las sugerencias que prometen el desarrollo son las mismas que lo inhiben.

De acuerdos a Sturgeon y Memedovic (2011) la integración de las firmas seguidoras en las cadenas de valor puede desencadenar una bipolaridad de escenarios, creados a partir de la diferenciación de estándares al interior de la cadena y fuera de ella; además de imponer barreras en el aprendizaje que conduzcan al desarrollo desigual; por otro lado, existe considerable evidencia de que las rentas son acaparadas de manera creciente por las firmas líderes, especialmente aquellas ubicadas en la producción de tecnologías núcleo y control de marcas y diseño (Chesbrough, 2001; Gawer y Cusumano, 2002; Linden y Somaya, 2003 en Sturgeon y Memedovic, 2011: 4).

Las firmas seguidoras ganan menos, tienen menores salarios y son más sensibles al ciclo económico por la escala de la producción y el capital fijo; además, si estas firmas tienden a dominar un país o región en específico, se corre el riesgo de impactar profundamente el sistema de negocios sobre largos periodos que pueden encadenar a las firmas o industrias de un espacio específico a actividades de bajo valor en la red (Kawakami, 2011; Schmitz, 2004; Kawakami y Sturgeon, 2011 en Sturgeon y Memedovic, 2011: 4).

La sugerencia general en este contexto, es reconocer las diferencias, asimetrías y oportunidades que brinda la creciente atomización de la producción de mercancías y servicios, y construir una estrategia de integración a partir de proveer mercancías y servicios funcionales a la articulación de la red, que en un escenario de suma positiva, permitan proceso de aprendizaje y desarrollo que tengan como resultado el incremento en la participación de la ganancia total.

El obstáculo para seguir dichas recomendaciones son los patrones de competencia desigual entre líderes -mercados monopólicos u oligopólicos- y seguidores -mercados abiertos o semiabiertos- a través del establecimiento de técnicas y estándares como condición de competitividad. 
De acuerdo con la metodología propuesta en la primera parte de este documento, es la innovación la causa material del proceso de valorización e interdependencia entre los capitales. Si consideramos esto como cierto, y se recupera el supuesto de que el acceso a la innovación es limitado por los derechos de propiedad, los capitales involucrados en la competencia, en la cadena de valor, presentan tasas de ganancia desiguales explicadas por las diferentes condiciones concretas de producción. El capital líder, es aquel que produce en las mejores condiciones como consecuencia de la propiedad y/o control sobre la innovación. Lo que esperaríamos en el largo plazo es una nivelación de las tasas de ganancia vía la difusión de la innovación, por medio de la competencia como lo establecimos hacia al final del apartado anterior.

Lo que ocurre concretamente es que dicha difusión se encuentra perturbada por "barreras a la entrada" que la retrasan, pero no eliminan. De esta manera los patrones de competencia en la nivelación de la tasa de ganancia se afectan por una inercia de carácter extraeconómico. ${ }^{33}$ Los capitales produciendo en las mejores condiciones protegen su propiedad a partir de diversas herramientas institucionales que impiden su difusión, retrasando así el progreso. ${ }^{34}$ En este escenario en la búsqueda por la nivelación de las ganancias se establecen diferentes patrones de competencia que explican la distribución de la ganancia y su falta de nivelación.

Lo anterior, no obstante, no suprime el mecanismo presentado en la metodología de este documento. La innovación no puede ser encapsulada permanentemente. Bajo diversas estrategias de copia, imitación, adaptación, entre otras, el segmento monopolizado se abre paulatinamente y se difunde, abriendo nuevas oportunidades para nuevos capitales, expandiendo/constriñendo así la cadena de valor. Bajo este análisis, la causa del desarrollo de los capitales complementarios, es en primera instancia su capacidad de competir, imitando al líder o innovando al desplazarlo. Las dificultades del primer caso dependerán de la fortaleza de los instrumentos institucionales que protegen el monopolio.

Bajo esta perspectiva el cumplimiento de los estándares no se definen como condición del desarrollo, sino como instrumentos de política, privada o pública, como consecuencia de la competencia entre capitales en un contexto de tasas de ganancia desiguales; son en última instancia mecanismos para

\footnotetext{
33 34 Exceptuando el caso del monopolio natural.

El progreso capitalista es la tendencia a superar históricamente las condiciones materiales de producción que permitan una creciente acumulación de capital.
} 
sostener la posición de monopolio del líder y/o transmisores de información entre líderes y seguidores. Cumplirlos no es condición necesaria del desarrollo. Sin embargo, sí han llegado a erigirse como condición de competitividad más allá de la lógica del proceso de acumulación.

Sólo aquello que permita imitar o superar las mejores condiciones de producción, tendrá como consecuencia un incremento en la participación de la ganancia de acuerdo al planteamiento aquí desarrollado. La gobernanza en la cadena de valor expresa la capacidad de inhibir la difusión de la innovación por parte de quien la posee.

Las cadenas de valor en este sentido son la configuración organizacional a la expansión y autonomización del capital, y no su causa. La competencia entre capitales que éstas expresan es consecuencia del desarrollo ocasionado por la innovación como driver of development a partir de la cual se amplía y especializa el espacio de reproducción del capital.

\section{CONCLUSIONES}

Las aportaciones que ha introducido el marco analítico de las cadenas globales de valor, son significativas en importancia en el diálogo académico en la medida en que han permitido descubrir, gracias a una gran riqueza de análisis empíricos, las nuevas formas que adopta la organización de los capitales, ahora regionales y globales, como expresión de la competencia a partir de los cambios históricos que ocasionó la revolución en la informática y las telecomunicaciones.

Una de las preocupaciones iniciales del marco de la cadena de valor era, y lo sigue siendo, la necesidad de "mapear" los proceso de producción y circulación en orden de medir con nuevas herramientas el comercio internacional -global e interdependiente-, el cual, oculta sus datos reales tras las categorías obsoletas de exportaciones e importaciones. ${ }^{35}$ Los esfuerzos en este sentido se 
han encaminado a diferenciar dichas categorías por tipo de bien y flujo, intra firma o inter firma.

En un contexto de competencia global altamente integrado e hiperespecializado, las consecuencias en el contagio de las perturbaciones crece, al tiempo que los flujos de información se amplían, sin contar todavía con las herramientas que nos permitan seguirlos y medirlos. Ser competitivo en esta nueva realidad, implica en primera instancia ser atractivo para los capitales globales que dirigen el comercio mundial. Las estrategias que conducen a lo anterior pueden tener efectos positivos en los capitales individuales si nos ubicamos en el plano de análisis de la firma, no obstante, arrojan diferentes resultados cuando consideramos el fenómeno en general, pues lo que es "bueno" para el capital individual, no necesariamente lo es para el conjunto. Es en este punto en el que quiero centrar la atención del lector.

Gran parte de los planteamientos generales que se realizan a partir del marco de la cadena de valor, son resultado de lecciones de diversos casos de estudio que arrojan "regularidades" que se han esquematizado en conceptos como la gobernanza o el ascenso industrial (Gereffi et al., 2005). La suma de estas experiencias se ha interpretado como diagnóstico general, haciendo equivalentes al todo, la suma de las partes, y llevando a consideraciones que se sintetizan en una gran estrategia de integración y ascenso como base para discutir el desarrollo.

Retomando la lógica de la metodología aquí expuesta, el desarrollo del capitalismo sólo es posible en la medida en que el proceso de acumulación a través del ciclo del capital se extiende e intensifica, produciendo a través de medios cada vez más productivos. Si en este contexto suponemos que el ascenso está en función de la subordinación de unos capitales por otros, estamos invirtiendo toda la lógica del sistema, confundiendo los síntomas con las causas, ya que dicha subordinación es la consecuencia de la competencia, sobre la base de diversos métodos de producción con diversas productividades.

Obviando lo anterior, lo que el marco de las cadenas de valor hace, es crear herramientas de política pública y privada que funcionalicen dicha subordinación, recrudeciéndola temporalmente, al tiempo que retrasan los proceso de nivelación en la distribución de los beneficios que sólo pueden alcanzarse por la creciente movilidad del capital y la fuerza de trabajo, es decir, vía la liberalización de los mercados de capital y trabajo gracias a medios cada vez más productivos.

on the sector in C producing intermediate goods for A". http://www.oecd.org/sti/ind/whatistradeinvalueadded.htm 
Esta liberalización permite en teoría, que los flujos de información se liberen y difundan con mayor rapidez nivelando la tasa de ganancia ${ }^{36}$ y acelerando la evolución económica. Esta tesis supone, sin embargo, "la total libertad del comercio... la eliminación de todos los monopolios... el desarrollo del sistema crediticio... y la subordinación de las diversas esferas de la producción a los capitalistas... gran densidad de población... derogación de todas las leyes que impiden a los obreros trasladarse de una esfera de producción a otra... la indiferencia del obrero con respecto al contenido de su trabajo..." . ${ }^{37}$ La brecha con respecto a la realidad económica, nos permite observar que toda perturbación a dichos supuestos es un retraso en el desarrollo capitalista.

La cadena de valor, como expresión de la competencia y el salto histórico en la movilidad de capital, se presenta como síntoma del proceso de acumulación, ahora regional y global. Considerarla como plataforma del desarrollo sería un error metodológico que llevaría a consideraciones poco precisas respecto del rumbo del conjunto de los capitales. En este sentido, atender indiscriminadamente las sugerencias de política podría ocasionar que el beneficio que arroja en lo particular, sea revertido en lo general.

Entender desde la perspectiva económica el motor de la acumulación en las cadenas de valor, posiciona a la innovación como la variable central en la discusión del desarrollo, sin embargo, dado que las condiciones del modelo arriba señaladas no se cumplen, los aspectos institucionales que conducen el desarrollo o la inhibición de ésta se consideran relevantes, es decir, viniendo de regreso en el argumento, los diferentes indicadores, manuales y políticas sugeridas por el marco de las cadenas de valor podrían alegarse vigentes, si y sólo si, en el largo plazo estimulan la liberalización del capital y del trabajo expandiendo el espacio de reproducción, lo cual podría en un lapso más corto, entrar en contradicción con consideraciones de bienestar social.

Los temas pendientes son variados, sobre todo, en los aspectos concretos de los casos de estudio. Se espera que el ejercicio aquí presentado contribuya a enriquecer la discusión y permita subsecuentes desarrollos. nancia derivada del valor producido a lo largo de la red en cuestión. 


\section{BIBLIOGRAFÍA}

Bair, Jennifer (2005), Global Capitalism and Commodity Chains: Looking Back, going Forward, Competition \& Change, vol. 9 issue 2, pp.153-180. Gereffi, Gary (2001), "Las cadenas productivas como marco analítico para la globalización”, Problemas del Desarrollo, vol. 32, núm. 125, México, IIECUNAM, abril-junio, pp. 9-37.

(2001a), "Shifting Governance Structures in Global Commodity Chains, with Special Reference to the Internet", American Behavioral Scientist, vol. 44, núm. 10, junio, pp. 1616-1637.

(2005a), "The Global Economy: Organization, Governance, and Development, en Neil J. Smelser y Richard Swedberg (eds.), The handbook of economic sociology, Ed. Princeton University, pp.160-182, New York.

Gereffi, Gary, John Humphrey y Timothy Sturgeon (2005), "The Governance of Global Value Chains", Review of International Political Economy, vol. 12, núm. 1, febrero, pp. 78-104.

Gereffi, Gary y Miguel Korzeniewicz (1994), Coomodity Chains and Global Capitalism, London, Ed. PRAEGER.

Kaplinsky, Raphael (1998), Globalisation, Industrialisation and Sustainable Growth: The Pursuit of the Nth Rent" Discussion Paper, núm. 365, Brighton, Institute of Development Studies, University of Sussex.

Marx, Carlos (1976 [1894]), El capital. El proceso global de la producción capitalista, tomo III, vol. 6, México, Siglo XXI Editores, pp. 431.

(1976 [1894]), El capital. El proceso de circulación de capital, tomo II, vol. 4, México, Siglo XXI Editores, pp. 428.

(1976 [1894]), El capital. El proceso de producción del capital, tomo I, vol. 1, México, Siglo XXI Editores, pp. 381.

Oliver E. Williamson (1981), "The Economics of Organization: The Transaction Cost Approach", The American Journal of Sociology, núm. 87, vol. 3, November, pp. 548-575.

Pérez, Carlota (2004), Revoluciones tecnológicas y capital financiero, la dinámica de las grandes burbujas financieras y las épocas de bonanza, México, Siglo XXI Editores.

Sandoval, Seyka (2013), La cadena global de hortalizas. La estrategia de ascenso de los productores sinaloenses, México, IIEC-UNAM.

Schumpeter, Joseph A. (1997 [1912]), Teoría del desenvolvimiento económico, México, Fondo de Cultura Económica, pp. 255.

(2002 [1939]), Ciclos económicos. Análisis teórico, histórico y estadístico del proceso capitalista, Zaragoza, Prensas Universitarias de Zaragoza, Edición Kindle. 
Sturgeon, T. y C. Memedovic (2011), Mapping Global Value Chains: Intermediate Goods Trade and Structural Change in the World Economy, Working Paper 05/2010.

\section{REFERENCIAS ELECTRÓNICAS}

Center on Globalization, Governance \& Competitiveness. Projects (2015) (consultado el 10 de abril de 2015) disponible en http://www.cggc.duke. edu/projects.php Research Reports, Publications and Presentations: 2009-2014 (2015) (consultado el 10 de abril de 2015) disponible en http://www.cggc.duke. edu/pdfs/CGGC-Reports-Pubs-Presentations.pdf

IMF, Trade Interconnectedness: The World With Global Value Chains (2013) (consultado el 10 de abril de 2015) http://www.imf.org/external/np/pp/ eng/2013/082613.pdf

OeCD, What is Trade in Value added? Why is it Important? (2015) (consultado el 10 de abril de 2015) disponible en http://www.oecd.org/sti/ind/ global-value-chains.htm

UNCTAD, Search (2015) (consultado el 10 de abril de 2015) http://unctad.org/ SearchCenter/Pages/Results.aspx?k=global\%20value\%20chains,

World Trade Organization, "Made in the World" (2015) (consultado el 10 de abril de 2015) disponible en https://www.wto.org/english/res_e/statis_e/ miwi_e/miwi_e.htm 